El consumo de radio y televisión de los estudiantes universitarios del Ecuador

Abel Suing, Kruzkaya Ordóñez y Carlos Ortiz

Question, Vol. 1, N. 57, e028, enero-marzo 2018. ISSN 1669-6581

http://perio.unlp.edu.ar/ojs/index.php/question/article/view/4420

FPyCS | Universidad Nacional de La Plata

La Plata | Buenos Aires | Argentina

Recibido: 19-10-2017 Aceptado: 14-11-2017

Cita sugerida: Suing, A., Ordóñez, K., y Ortiz, C. (2018). El consumo de radio y televisión de los estudiantes universitarios del Ecuador. Question, 1(57), e028. doi:https://doi.org/10.24215/16696581e028

\title{
El consumo de radio y televisión de los estudiantes universitarios del Ecuador
}

The consumption of radio and television of the university students of the Ecuador

\author{
Abel Suing, Kruzkaya Ordóñez y Carlos Ortiz \\ Universidad Técnica Particular de Loja (Ecuador) \\ arsuing@utpl.edu.ec; kordonez@utpl.edu.ec; ccortiz@utpl.edu.ec
}

\section{Resumen}

El propósito de la investigación es conocer las tendencias de consumo audiovisual de los estudiantes universitarios del Ecuador entre 2015 y 2016. La metodología utilizada es la cuantitativa y cualitativa a través de una encuesta nacional, sondeos y entrevistas. Las hipótesis giran en torno al uso simultáneo de las redes sociales, mientras los jóvenes ven televisión o escuchan radio, la correlación entre consumos de medios e Internet y la incidencia de los dispositivos de acceso a la red. Se concluye que, los medios tradicionales, televisión abierta, de pago, la radio son sustentables en el tiempo. El Internet no supone su desaparición pero, condiciona a que los medios se adapten a una estructura de comunicación dual, física y digital, fundamental para su existencia. 
Palabras clave: televisión; consumo televisivo; jóvenes; internet; redes sociales.

\section{Abstract}

The purpose of the research is to know the audiovisual consumption trends of university students from Ecuador between 2015 and 2016. The methodology used is quantitative and qualitative through a national survey, surveys and interviews. The hypotheses revolve around the simultaneous use of social networks, while young people watch television or listen to radio, the correlation between media consumption and the Internet and the incidence of network access devices. It is concluded that, traditional means, open television, television of pay, radio are sustainable over time. The Internet does not suppose its disappearance but, it conditions to that the means adapt to a structure of dual communication, physical and digital, fundamental for its existence.

Key words: television; television consumption; young, internet; social networks.

Quienes integran la "i-Generación" (Marta, Martínez y Sánchez, 2013: 41) denominados también "nativos digitales" (Prensky, 2001) han crecido interactuando con grupos de diversas partes del mundo gracias al acceso a Internet, "nos guste o no, aquí es donde nuestros adolescentes hablan, traman, ríen y pelean con algunas de las personas más importantes en sus vidas" (Gómez, 2015).

Los nativos digitales viven una época de cambios en las formas de alcanzar el conocimiento no conocidas hasta ahora (Barrios, 2013; Cabero, 1994), por ejemplo en el paso de viejos a nuevos medios de comunicación sin que ello signifique el abandono definitivo de los medios tradicionales. La prensa, radio y televisión continúan proporcionando información a los jóvenes (Rivière, 2003; Masterman, 2010).

Con la presencia de Internet parecería que la influencia de los medios en los jóvenes es mínima, sin embargo para los adolescentes aún "resulta inconcebible un mundo sin televisión" (Sesento, 2015). La acogida de los medios tradicionales se refleja en la televisión que en ocho décadas de emisión se ha convertido en "el medio de comunicación más influyente para el desarrollo de patrones de comportamiento de las audiencias; los niños y adolescentes del mundo entero han crecido conjuntamente con la evolución del mercado televisivo" (Sandoval, 2006: 206). La televisión pretende "dar respuesta a una gran diversidad de necesidades básicas, desde las cognitivas a las de entretenimiento" (Zamora, 2004: 7). 
El desarrollo de las tecnologías audiovisuales incide en el cambio de la forma de ver televisión, de la tradicional reunión familiar a la recepción individual (Rubio, 2010: 208) aunque los jóvenes hayan migrado a Internet, la televisión continúa estableciendo formas de consumo (Cáceres, Ruiz y Brändle, 2011; Marth-Lazo y Gabelas-Barroso, 2013), lo que se programa en las nuevas vías de comunicación no se aparta de la tradicional emisión televisiva (Menor, 2010) además el tránsito hacia nuevas formas de consumo no está acompañado de una renovación en los contenidos.

Parece que los jóvenes no están frente al "fin de la televisión como medio de comunicación, sino ante el final de un determinado uso o consumo a través de una determinada pantalla" (Fernandez-Planells y Figueras-Maz, 2012: 196), apreciación apoyada en la "migración de los más jóvenes a servicios de streaming como Netflix [...] fenómeno que coloca a la televisión tradicional frente a un panorama sombrío de cara al futuro" (TtvNews, 2014) sin embargo deja claro que la digitalización televisiva e Internet han cambiado la forma de concebir la televisión (Zuberogoitia et al., 2016).

De manera simultánea al fenómeno de la televisión en Internet ocurre la interacción entre televidentes en redes sociales hecho evidente al conocer que en 2016 Facebook y YouTube superaron a la televisión como fuente primaria de acceso a la información entre los jóvenes. Según el informe de The Reuters Institute for the Study of Journalism los medios sociales se consolidan como espacios para consumir noticias (Newman et al., 2016).

A nivel regional la Comisión Económica para América Latina de las Naciones Unidas señala que los sitios de Internet más populares son las redes sociales. Los usuarios se conectan mayoritariamente a Google, Facebook, Microsoft, Yahoo y Wikipedia. Facebook es la red social con mayor audiencia que copa el $95 \%$ del tiempo dedicado a medios sociales, además el uso de redes sociales no está directamente vinculado a los niveles de ingreso (CEPAL, 2015).

La interacción en redes sociales comentado lo que se ven en televisión ocurre a través de varias pantallas, "varios estudios confirman que el consumo y la interacción que los jóvenes desarrollan alrededor de las pantallas (televisión, videojuegos, Internet, móviles) son un factor socializador de primer orden [...] colaboran en la construcción de una cultura digital" (Barroso, 2011: 84), es decir "Internet se ha convertido en fuente de entretenimiento y socialización" (Fernandez-Planells y Figueras-Maz, 2012: 177) por lo que determinar las relaciones entre televisión y redes sociales puede ser un elemento que describa un nuevo perfil de consumidores.

Investigaciones realizadas entre 2005 y 2015 centradas en los hábitos de consumo de televisión entre jóvenes y estudiantes universitarios, hombres y mujeres, de hasta 25 años de edad, de España, Ecuador, Colombia y Perú, de las Universidades de Málaga, Complutense de Madrid, Rey Juan Carlos, Carlos III, Zaragoza, UTPL y Universidad de Manizales (ArangoForero y González-Bernal, 2009; Arango-Forero, 2008; Cáceres, Ruiz y Brändle, 2011; 
Cordicom, 2015; Fernandez-Planells y Figueras-Maz, 2012; Gómez, López y Martín, 2011; Marín, Yaguana y Barrazueta, 2013; Marth-Lazo y Gabelas-Barroso, 2013; Martínez, 2005; Vergara et al., 2009), determinan las siguiente características del uso actual que los jóvenes dan a la televisión:

-Los estudiantes universitarios en Iberoamérica utilizan la televisión para entretenerse e informarse.

-Los contenidos preferidos son películas, videos musicales, series, magazines, programas del corazón y reality shows.

-Cuando emplean la televisión como medio de información prefieren los programas de actualidad, los documentales y los noticieros.

-Los programas de opinión y de farándula son los menos apreciados, consideran que hay exceso de periodismo rosa.

-Los jóvenes prefieren las televisiones privadas sobre las públicas cuya señal reciben gracias a la alta penetración de televisión por suscripción.

-La casa es el lugar predilecto para ver televisión sin embargo el visionado en familia ha dejado espacio al consumo individual desde la habitación.

-La noche es momento preferido para ver televisión.

-De lunes a viernes consumen más televisión nacional pero durante el fin de semana migran a la oferta internacional.

-Los jóvenes prefieren canales variados sobre los temáticos pero manifiestan preocupación porque no encuentran constantes ofertas culturales y hay pocos espacios cinematográficos serios.

En Ecuador de acuerdo el estudio "Los hábitos digitales de los ecuatorianos" (Netlife, 2015) Internet influye en las prácticas de los medios de comunicación y modifica la vida de las personas lo que se refleja en los siguientes indicadores: $79 \%$ de los usuarios considera que lo que más hacen por Internet es comunicarse con otras personas, $71 \%$ ven televisión y navegan por Internet al mismo tiempo, 47\% visitaron los sitios Web anunciados en televisión; los autores del estudio señalan que "los resultados configuran nuevos hábitos para más de 5.5 millones de ecuatorianos con lo cual es indudable que el acceso a Internet está cambiando la forma de vida en el Ecuador". Otra evidencia de la incorporación de las redes sociales en la vida cotidiana es que:

De los 8,5 millones de usuarios de Internet en Ecuador, Facebook continúa siendo el líder absoluto habiendo captado a 8,1 millones de usuarios [....] [Twitter] cuenta con un promedio de 2000.000 de usuarios [...] de los cuales el $53 \%$ en promedio utiliza la red social desde dispositivos móviles (Del Alcázar, 2015). 
Los contenidos preferidos por los jóvenes ecuatorianos coinciden con "gustos similares a los de otros países: comedias, ficción-acción, dibujos animados, música y deportes, principalmente" (MINTEL, 2011). En las principales ciudades de Ecuador, Quito y Guayaquil, los medios de comunicación empleados para acceder a información son televisión, Internet, radio y periódicos. Para los adolescentes ecuatorianos de 16 a 17 años su mayor fuente de información es Internet, luego la televisión (Fundamedios, 2013).

En 2016 los jóvenes de 15 a 19 años representan el 9,40\% de la población de Ecuador (INEC, 2016) y cerca de dos tercios de ellos "acuden a la televisión como el principal medio de comunicación para informarse (...) De forma general las tendencias son televisión, Internet, prensa escrita, radio" (Cordicom, 2015: 6).

Por lo expuesto se estima importante establecer las tendencias de consumo audiovisual de los jóvenes universitarios de Ecuador porque permitirán conocer la relación entre los consumos de radio y televisión y las nuevas prácticas en redes sociales de la generación de nativos digitales, además se identificarán las demandas de los profesionales en formación cercanos a insertarse en el sector productivo de una sociedad cada día más imbuida en lo digital.

Los adolescentes y los jóvenes son objeto de estudio interesante de analizar por su fácil adaptación y respuesta a los cambios, por su permanente deseo de exploración, clasificación y decantación del medio, y porque son ellos hoy los adultos consumidores de mañana (ArangoForero, 2008: 14).

Los jóvenes universitarios constituyen un grupo de interés en tanto han crecido en entornos digitales de interacción, "han tenido, tienen y tendrán acceso a las nuevas tecnologías, aspecto que les dota de claras competencias mediáticas a la hora de interactuar con las nuevas tecnologías" (García y Díaz, 2012: 106).

Los objetivos de la investigación son: 1. Identificar las formas de consumo de televisión y de radio así como el empleo de medios sociales por parte de los jóvenes universitarios de Ecuador entre 2015 y 2016 y 2. indagar las razones que expliquen las tendencias de consumo de radio y televisión por parte de los jóvenes universitarios de Ecuador.

Las hipótesis son:

1. Los jóvenes universitarios del Ecuador:

a. Consumen poca televisión abierta, prefieren la televisión de pago, y en ambas consumen más contenidos generalistas y de entretenimiento.

b. Hacen uso frecuente de redes sociales mientras consumen medios tradicionales de comunicación.

c. Consumen radio a través de emisiones digitales.

2. No existe correlación entre los consumos de radio y televisión con el acceso a Internet. 
3. Los dispositivos de acceso a Internet inciden en el consumo de medios de comunicación tradicionales e influyen en la adaptación de la oferta de contenidos.

\section{Metodología}

La metodología empleada es cuantitativa y cualitativa. La información cuantitativa proviene del proyecto de investigación nacional "Consumo y usos de medios de comunicación en estudiantes universitarios en tiempos de convergencia" desarrollado por el Departamento de Ciencias de la Comunicación de la Universidad Técnica Particular de Loja (UTPL) que involucró a 25 de las 52 universidades existentes en Ecuador. La muestra se calculó de acuerdo a la fórmula estadística para poblaciones finitas con un $98 \%$ de confianza y un $2 \%$ de margen de error, se realizaron 8.217 encuestas en el período abril-agosto de 2015, se consideró también las categorías de universidades A, B y C que establece el Consejo de Evaluación, Acreditación y Aseguramiento de la Calidad de la Educación Superior del Ecuador. 


\begin{tabular}{|c|c|c|c|c|}
\hline $\mathbf{N}^{\circ}$ & Universidades & Categoría & Población & Muestra \\
\hline 1 & Escuela Politécnica del Litoral & A & 9.700 & 370 \\
\hline 2 & Escuela Politécnica Nacional & A & 10.000 & 370 \\
\hline 3 & ESPOCH & $B$ & 16.902 & 376 \\
\hline 4 & PUCE & $B$ & 10.688 & 371 \\
\hline 5 & Católica de Cuenca & $\bar{D}$ & 10.315 & 370 \\
\hline 6 & Central del Ecuador & B & 32.426 & 380 \\
\hline 7 & De las Américas & B & 24.402 & 378 \\
\hline 8 & De los Hemisferios & B & 673 & 245 \\
\hline 9 & Del Azuay & B & 1.500 & 362 \\
\hline 10 & Estatal Amazónica & B & 571 & 230 \\
\hline 11 & Estatal de Bolívar & C & 2.962 & 340 \\
\hline 12 & De Cuenca & A & 30.000 & 379 \\
\hline 13 & Iberoamericana del Ecuador & B & 451 & 208 \\
\hline 14 & Internacional del Ecuador & B & 2.124 & 325 \\
\hline 15 & Laica Eloy Alfaro de Manabí & D & 16.268 & 375 \\
\hline 16 & Metropolitana UMET & C & 1.071 & 283 \\
\hline 17 & Nacional de Loja & $B$ & 19.383 & 377 \\
\hline 18 & San Gregorio de Portoviejo & $\mathrm{C}$ & 2.635 & 335 \\
\hline 19 & Politécnica Estatal del Carchi & B & 746 & 254 \\
\hline 20 & Regional Amazónica Ikiam* & & 148 & 107 \\
\hline 21 & San Francisco de Quito & A & 13.225 & 373 \\
\hline 22 & Técnica de Ambato & B & 16.610 & 375 \\
\hline 23 & Técnica Particular de Loja & B & 6.073 & 361 \\
\hline 24 & Tecnológica Equinoccial & $B$ & 6.250 & 362 \\
\hline \multirow[t]{2}{*}{25} & Tecnológica Israel & C & 1.630 & 311 \\
\hline & Total de la muestra & & & 8.217 \\
\hline
\end{tabular}

Tabla1. Conformación de la muestra. Fuente: Consejo de Educación Superior de Ecuador.

*La Universidad Regional Amazónica se creó en 2013 y aún no está categorizada. Elaboración propia.

Para contrastar los datos y sobre la base de herramientas metodológicas cualitativas se consultó las preferencias de consumo audiovisual a través de un sondeo aplicado a 128 estudiantes de 14 universidades, del 2 de marzo al 11 de mayo de 2016. También se realizaron 10 entrevistas semi-estructuradas a expertos en la producción y emisión audiovisual del Ecuador entre el 18 y 29 de abril de 2016 (tabla 2). 


\begin{tabular}{|c|c|c|c|}
\hline $\mathbf{N}^{\circ}$ & Nombre & Estación/Cargo & Observación \\
\hline 1 & $\begin{array}{c}\text { Eulalia } \\
\text { Eguiguren }\end{array}$ & $\begin{array}{c}\text { Tele-amazonas/Gerente } \\
\text { de Producción }\end{array}$ & $\begin{array}{c}\text { Televisión de propiedad } \\
\text { privada }\end{array}$ \\
\hline 2 & $\begin{array}{l}\text { Sebastián } \\
\text { Tandazo }\end{array}$ & RTS/Productor Combate & $\begin{array}{c}\text { Televisión de propiedad } \\
\text { privada }\end{array}$ \\
\hline 3 & Viko Valdivieso & $\begin{array}{l}\text { Ciudadano } \\
\text { TV/Productor }\end{array}$ & Televisión gubernamental \\
\hline 4 & Eddie González & $\begin{array}{c}\text { Ecuavisa/Director } \\
\text { creativo }\end{array}$ & $\begin{array}{c}\text { Televisión de propiedad } \\
\text { privada }\end{array}$ \\
\hline 5 & Renán Camacho & $\begin{array}{c}\text { TC- Quito/Gerente de } \\
\text { ventas }\end{array}$ & $\begin{array}{c}\text { Televisión de propiedad } \\
\text { privada }\end{array}$ \\
\hline 6 & Jemmy Molina & $\begin{array}{c}\text { Ecuavisa/Asistente de } \\
\text { programación }\end{array}$ & $\begin{array}{c}\text { Televisión de propiedad } \\
\text { privada }\end{array}$ \\
\hline 7 & Alex Romero & $\begin{array}{c}\text { Canal Sur/Jefe de } \\
\text { noticias }\end{array}$ & $\begin{array}{c}\text { Televisión de propiedad } \\
\text { pública }\end{array}$ \\
\hline 8 & Gonzalo Ojeda & $\begin{array}{l}\text { Radio Super } \\
\text { Láser/Gerente }\end{array}$ & Radio de propiedad privada \\
\hline 9 & Xavier Espinoza & Radio Gaviota/Gerente & Radio de propiedad privada \\
\hline 10 & Diego Ortiz & $\begin{array}{c}\text { Exa Ibarra/Coordinador } \\
\text { General }\end{array}$ & Radio de propiedad privada \\
\hline
\end{tabular}

Tabla 2. Perfiles de entrevistados. Fuente: elaboración propia

\section{Resultados}

De acuerdo a los resultados cuantitativos el consumo de los medios de comunicación convencionales por parte de los jóvenes universitarios del Ecuador no es frecuente. La televisión está presente en el consumo cotidiano en una tercera parte de los estudiantes. Los contenidos que predominan son ficción y música tanto en televisión como en radio, en la figura 1 se muestran las preferencias en una escala cualitativa de 1 a 5 , en donde 1 es la mayor preferencia, se observan inclinaciones simultáneas para los contenidos informativos y de deportes. 


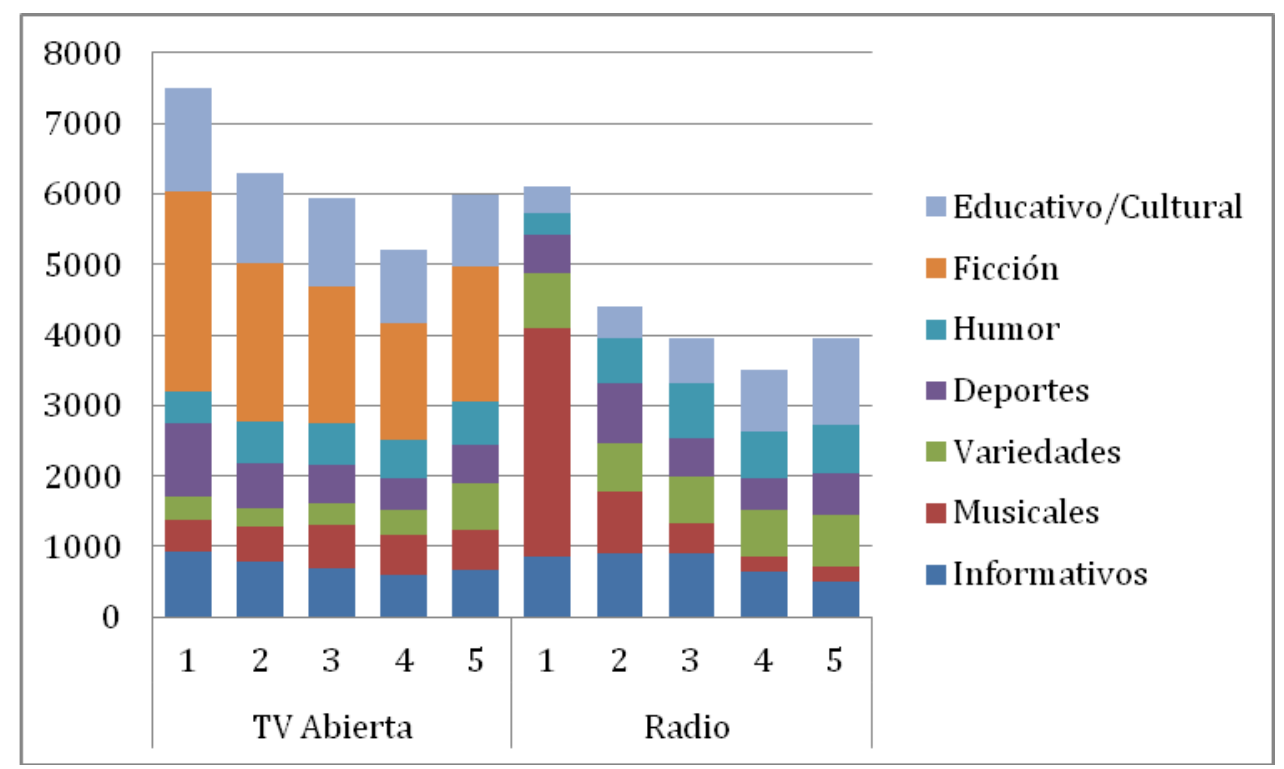

Figura 1: Preferencias en el consumo de televisión abierta y radio. Fuente: Departamento de Ciencias de la Comunicación de la UTPL.

La televisión de señal abierta es generalista en sus contenidos mientras que la televisión de pago es temática, en ésta se ubican estaciones dedicadas a públicos específicos por ello llama la atención que las estaciones de pago preferidas sean generalistas.

Hay elementos que permiten identificar una tendencia en el consumo de los medios audiovisuales y la integración de éstos en nuevos entornos y herramientas. Por un lado está el uso de Internet en el tiempo libre que tiene una proporción mayor al consumo de los medios de comunicación y por otro lado $30 \%$ de los estudiantes encuestados señalan que mientras ven televisión o escuchan radio también están en redes sociales, revisando sus correos o navegando en Internet. Un aspecto a destacar es que los consumos de radio ocurren en mayor medida mientras los jóvenes están en movimiento ya sea caminando o transportándose en vehículos gracias a la conectividad que ofrecen las nuevas tecnologías y la disponibilidad del espectro radioeléctrico (figura 2). 


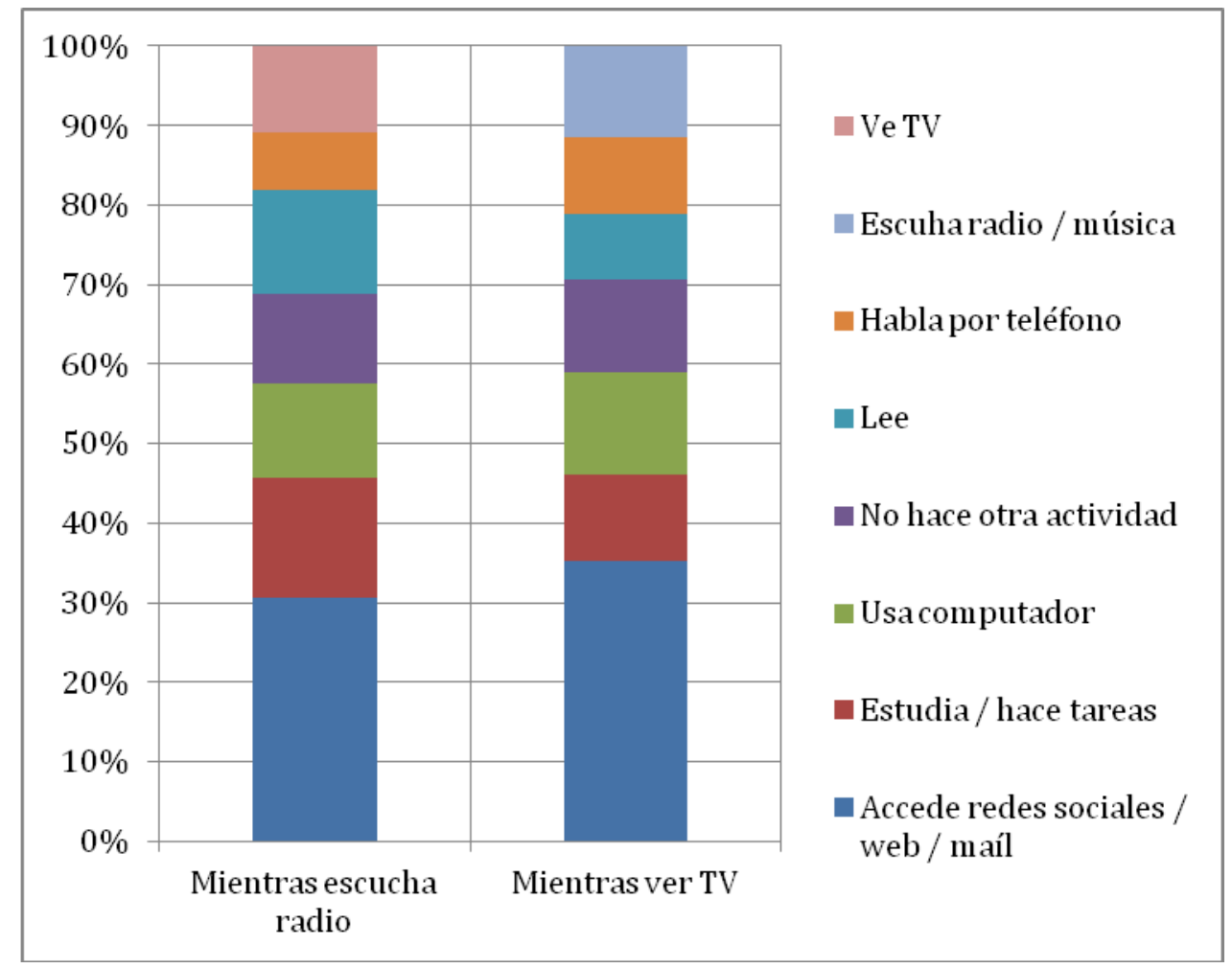

Figura 2. Actividades simultáneas. Fuente: Departamento de Ciencias de la Comunicación de la UTPL.

Para determinar la relación efectiva entre el consumo de televisión y radio con el acceso a Internet se realizó un análisis de correlación a través del coeficiente de correlación de rangos de Kendall (tau de Kendall), estadístico utilizado para medir la asociación entre variables ordinales, medida no paramétrica de asociación para variables ordinales. Las tablas 4,5 y 6 muestran la existencia de correlaciones altas de signo positivo, los niveles de significancia menores a 0.05 (bilateral) llevan a rechazar las hipótesis nulas, concluyendo que hay asociación entre las variables acceso a Internet y consumos de televisión abierta, de pago y radio. 


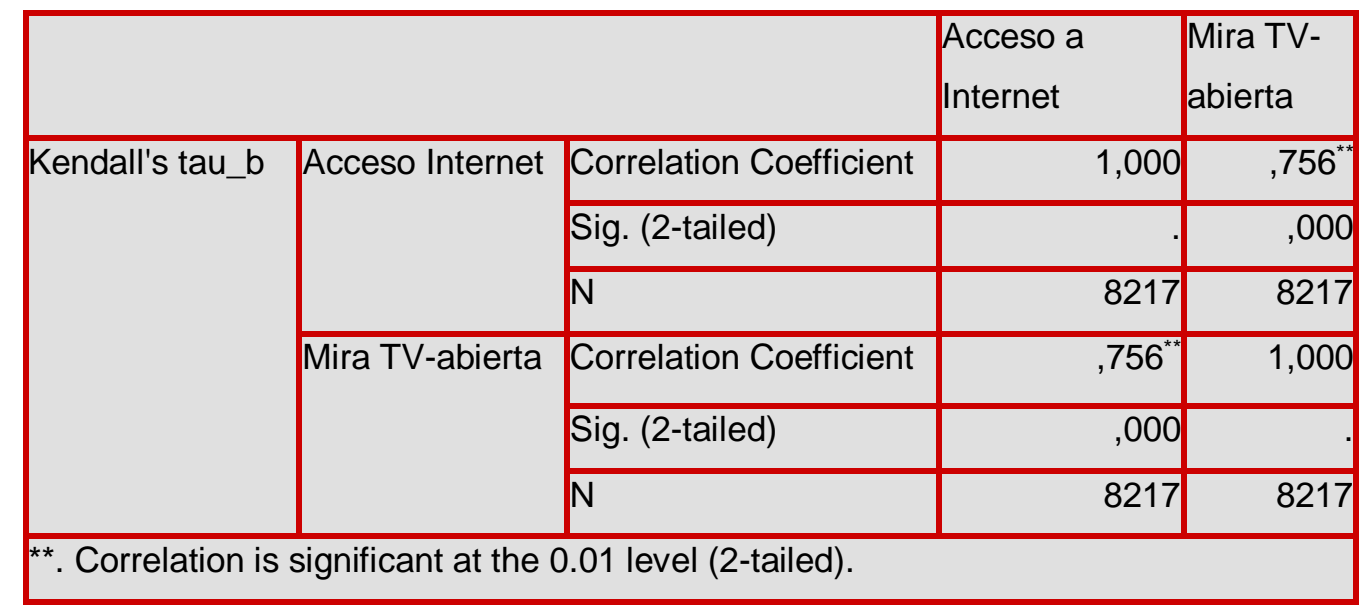

Tabla 3. Correlación entre acceso a Internet y TV-Abierta. Fuente: elaboración propia a través de SPSS Statistics versión 20.

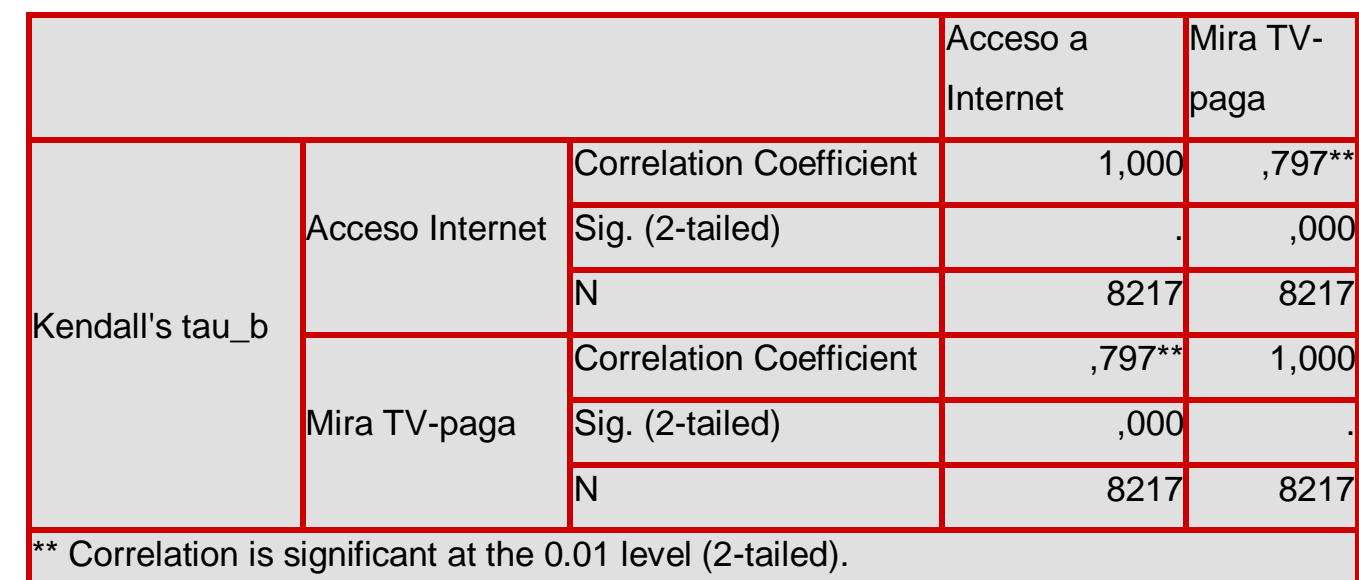

Tabla 4. Correlación entre acceso a Internet y TV de pago. Fuente: elaboración propia a través de SPSS Statistics versión 20.

\begin{tabular}{|c|c|c|c|c|}
\hline & & & $\begin{array}{l}\text { Acceso a } \\
\text { Internet }\end{array}$ & $\begin{array}{l}\text { Hábito de } \\
\text { escuchar radio }\end{array}$ \\
\hline \multirow{6}{*}{ Kendall's tau_b } & \multirow{3}{*}{$\begin{array}{l}\text { Acceso a } \\
\text { Internet }\end{array}$} & Correlation Coefficient & 1,000 &, $786^{* *}$ \\
\hline & & Sig. (2-tailed) & & ,000 \\
\hline & & $\bar{N}$ & 8217 & 8217 \\
\hline & \multirow{3}{*}{$\begin{array}{l}\text { Hábito de } \\
\text { escuchar radio }\end{array}$} & Correlation Coefficient &, $786 *+$ & 1,000 \\
\hline & & Sig. (2-tailed) & ,000 & \\
\hline & & $\mathrm{N}$ & 8217 & 8217 \\
\hline
\end{tabular}

Tabla 5. Correlación entre acceso a Internet y radio. Fuente: elaboración propia a través de SPSS Statistics versión 20. 
Los resultados del sondeo confirman las tendencias de consumo. Los jóvenes interactúan en redes sociales comentando lo que ven en televisión abierta y de pago, $17 \%$ en el mismo momento, $40 \%$ al concluir los programas; y, 43\% nunca interactúan. Las redes sociales preferidas son: Facebook (55\%); WhatsApp (14\%); YouTube (13\%); Instagram (9\%), Twitter (7\%) y Flickr (2\%).

Los jóvenes explican que las razones para interactuar en redes sociales mientras ven televisión son: compartir opiniones con otras personas sobre lo que están viendo $(49 \%)$, revisar correos electrónicos, web u otras redes sociales (36\%), votar o enviar comentarios a programas en vivo (13\%) y comprar (2\%). La interacción de los jóvenes de acuerdo al tipo de contenidos ocurre en programas de entretenimiento $(29 \%)$, informativos (17\%), formativos, educativos, culturales $(15 \%)$, deportivos $(12 \%)$, de opinión (8\%), publicitarios $(4 \%)$ y otros $(15 \%)$.

Respecto al visionado de televisión a través de Internet, $48 \%$ de los estudiantes señalan que sí lo hacen mientras que $52 \%$ dicen que no (figura 3). Quienes utilizan la red para ver televisión emplean diferentes dispositivos como smartphone (24\%), computadores portátiles (41\%), computadores de escritorio (10\%), tablets (3\%), smart TV (3\%), consolas (11\%) y otros equipos (8\%).

La mayoría de jóvenes consumen televisión a través de Internet menos de hora diaria. El tiempo dedicado por día para ver televisión a través de Internet fluctúa entre menos de una hora $(42 \%)$, una hora $(22 \%)$, dos $(25 \%)$, tres $(7 \%)$ y más de tres horas $(4 \%)$. Las razones para ver televisión por Internet de acuerdo a las respuestas del sondeo son: Independencia de horarios de programación (33\%), evitar la censura porque no hay restricciones contenidos (20\%), existe una amplia oferta de contenidos (19\%), comodidad, visionado individual (18\%) y consumo en simultáneo a otra actividades (10\%).

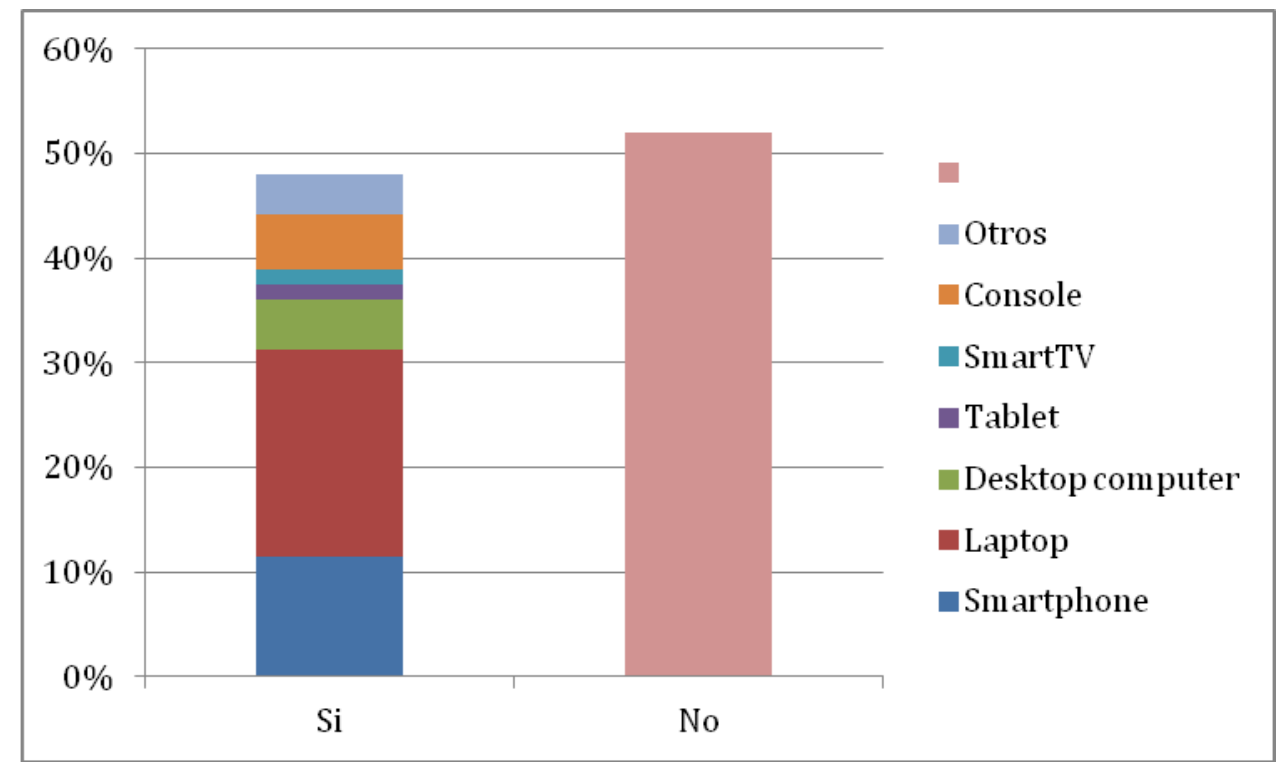

Figura 3. Visionado de televisión a través de Internet.

Fuente: Departamento de Ciencias de la Comunicación de la UTPL. 
Finalmente y de acuerdo a los expertos consultados los estudiantes universitarios consumen desde medios tradicionales hasta medios digitales, así mismo señalan que hay alta demanda de redes sociales en las cuales encuentran contenidos acordes a sus intereses y pueden estar en contacto con sus amigos. La presencia en redes sociales implicaría un decremento del consumo de televisión; sin embargo la industria televisiva no ve afectado su modelo de ingresos, ya que aún posee ratings importantes en telenovelas, películas y noticias.

Los estudiantes universitarios alcanzan cantidades significativas de información a través de Internet por esta razón las empresas televisivas despliegan estrategias digitales para no quedarse aisladas del conglomerado estudiantil. Desde la perspectiva de los expertos los medios trabajan en función de producción e ingresos, atienden prioritariamente al entretenimiento que es el género de mayor audiencia entre los jóvenes universitarios. Ante la irrupción de las redes sociales, la televisión tradicional como la televisión de pago se reinventarán frecuentemente y entre las medidas que adoptarán está el fortalecimiento de la producción local como un factor que marque la diferencia frente a los demás.

Frente al fortalecimiento de los contenidos locales que sugieren los expertos quizá vale recordar que la mayor diferencia entre televisión de señal abierta y de pago son los contenidos, en la segunda hay más programas dedicados a los jóvenes sin los incómodos espacios comerciales. La malla de contenidos de la televisión de pago denota altos presupuestos que la televisión local de señal abierta no puede cubrir, esta circunstancia justificaría que cada día se recurra al diálogo con las audiencias para de a poco integrar los hábitos de los jóvenes en los medios locales.

Las redes sociales amplifican la difusión de la televisión, permiten el diálogo, los medios de comunicación procuran añadir herramientas digitales ya que conocen que sus audiencias, particularmente los jóvenes, interactúan mientras visionan los contenidos. Si a la costumbre de los adolescentes de realizar varias actividades mientras está encendida la televisión se le agrega la dinámica de las redes sociales parecería que la televisión definitivamente queda relegada a un segundo plano. Para los jóvenes recibir la señal de televisión es remitirse a su teléfonos inteligentes, en estos encuentran incluso aquello que la televisión no transmite, es decir, no ven la televisión de la forma tradicional porque ya no la necesitan, no les interesa.

Respecto al futuro se estima que la era de la televisión tanto de pago como abierta no terminará ante el Internet porque la televisión convencional es la manera de socializar mediante la señal audiovisual. La televisión por Internet tiende a ser personalizada en cambio la televisión de señal abierta demanda de acuerdos entre los televidentes que comparten un lugar común. Para que la televisión abierta tenga mayor aceptación en los jóvenes debería presentar contenidos más atractivos, la televisión nacional ha vinculado los programas más exitosos a las 
redes sociales generando interés en los públicos digitales y que de esta forma vuelvan la mirada a la televisión tradicional.

La televisión por internet está generando una nueva demanda por la comodidad, busca nuevos métodos para llegar a la audiencia, especialmente a los jóvenes que prefieren contenidos más actuales acordes a sus intereses como la moda, estilos de vida, tendencias y estar en contacto con sus amigos.

En cuanto al consumo de radio llama la atención la cantidad de jóvenes que prefieren programas de diversión, escuchan más entretenimiento que información, se entiende que los dispositivos móviles les permiten estar informados, las redes sociales han postergado la demanda informativa en radio. Una causa probable del mayor consumo de entretenimiento es que los jóvenes desean olvidar los problemas de sus trabajos o centros educativos. Una forma de incrementar el consumo de información en radio serían las cápsula informativas, breves segmentos de hasta cinco minutos que permiten que los jóvenes estén informados mientras escuchan música.

La tendencia actual es que la radio en línea gana espacio a la radio analógica sin embargo para recibir una buena señal se necesita de amplio acceso a Internet que también significa alto costo. Por esta razón, según los expertos consultados, los medios de comunicación continuarán ocupado espacio radioeléctrico. La radio en línea es una buena herramienta para dar a conocer y expandir la programación pero pensar en una radio en línea al cien por ciento implicaría cambios completos de equipos tecnológicos, es decir parecería que la radio convencional no va a desaparecer en corto tiempo.

\section{Conclusiones}

Sobre la base de la información cuantitativa presentada en las tablas y figuras precedentes se señala que las primeras hipótesis se cumplen parcialmente, los jóvenes universitarios del Ecuador:

a. Consumen televisión abierta y aunque ven televisión de pago su recepción no es alta.

b. Consumen fundamentalmente contenidos generalistas y de entretenimiento en radio y televisión de señal abierta.

c. Hacen uso frecuente de Internet y redes sociales en su tiempo libre y a través de la red consumen medios tradicionales de comunicación.

d. Consumen radio sobre todo apoyados en las emisiones digitales y mientras están en movimiento, no escuchan radio de forma sedentaria. 
La segunda hipótesis se rechaza, sobre la base de la correlaciones de tau de Kendall presentadas en las tablas tres, cuatro y cinco se puede afirmar que existe correlación entre los consumo de radio y televisión con el acceso a internet. Por último y en relación a la información cuantitativa presentada en la figura tres se acepta la tercera hipótesis: los dispositivos de acceso a internet inciden en el consumo de medios de comunicación tradicionales e influyen en la adaptación de la oferta de contenidos.

El consumo de la televisión ya no es una experiencia universal ahora existe un sistema audiovisual con múltiples pantallas en cine, televisión abierta, de pago, satelital, video, Internet, celular; en este marco los jóvenes universitarios del Ecuador, según los resultados de la investigación, consumen televisión abierta en mayor proporción que la televisión de pago y la radio lo que significa que los medios tradicionales y en específico la televisión mantienen una posición importante en el mercado de los medios

La cultura audiovisual ecuatoriana es escasa, el televidente no acostumbra a consumir contenidos especializados por ello la oferta de televisión generalista en abierto o de pago es la de mayor aceptación, los jóvenes se inclinan a sintonizar programas de televisión nacional con los cuales se sienten identificados.

Los jóvenes universitarios del Ecuador consumen información en Internet de forma simultánea con la radio y televisión. Los jóvenes están navegando en Internet o revisando sus redes sociales mientras consumen televisión o radio.

El consumo de televisión abierta, de pago y la radio van de la mano con el internet, la audiencia se adapta cada vez a formatos de comunicación bidireccionales a través de las redes sociales como el Facebook, WhatsApp, Youtube, Instragram, Twitter. Los medios tradicionales, televisión abierta, de pago o la radio son sustentables en el tiempo, la existencia del Internet no supone su desaparición pero condiciona a que el conjunto de medios se adapten a una estructura de comunicación dual física y digital fundamental para su existencia.

La televisión por internet en Ecuador tiene acogida entre los jóvenes, el acceso a este servicio es cotidiano, los jóvenes permanecen conectados a través de varios dispositivos tecnológicos para observar, escuchar, opinar e interactuar con el mundo virtual, es adaptable a sus hábitos de consumo, a sus gustos y preferencias a diferencia de la televisión abierta que demanda acuerdos con los televidentes que comparten un lugar común.

\section{Bibliografía}

Arango-Forero, G. (2008). Fragmentación de audiencias en una sociedad multicanal: gustos y preferencias de adolescentes en Bogotá. Palabra Clave, 11(1), pp. 11-28. Recuperado de http://palabraclave.unisabana.edu.co/index.php/palabraclave/article/view/1412/1549 
Arango-Forero, G. y González-Bernal, M. (2009). Televidencias juveniles en Colombia : fragmentación generada por un consumo multicanal. Palabra Clave, 12(2), pp. 215234. Recuperado de http://palabraclave.unisabana.edu.co/index.php/palabraclave/article/viewArticle/1562

Barrios, A. (2013). Los jóvenes y la red; usos y consumos de los nuevos medios en la sociedad de la información y la comunicación. Signo y Pensamiento, 28(54), pp. 265-275.

Barroso, J. (2011). Screens and the youth in the augury of the new millennium [Pantallas $y$ jóvenes en el ágora del nuevo milenio]. Acimed, 22(1), pp. 79-90. Recuperado de http://www.scopus.com/inward/record.url?eid=2-s2.080051739223\&partnerID=40\&md5=c9b2d3cd9005592f473a341f4cf200a7

Cabero, J. (1994). Nuevas tecnologías, comunicación y educación. Comunicar, 3, pp. 14-25.

Cáceres, M.; Ruiz, J. y Brändle, G. (2011). El uso de la televisión en un contexto multipantallas: viejas prácticas en nuevos medios. Análisi, 43, pp. 21-44.

CEPAL (2015). La nueva revolución digital. De la Internet del consumo a la Internet de la producción. Santiago: Naciones Unidas.

Cordicom (2015). Inquietudes y propuestas de los jóvenes sobre la TV Ecuatoriana. Quito: Cordicom.

Del Alcázar, J. (2015). Ranking y Estadísticas Redes Sociales Ecuador. Formación Gerencial. Recuperado de http://blog.formaciongerencial.com/2014/05/16/ranking-redes-socialesecuador-mayo-2014/

Fernandez-Planells, A. y Figueras-Maz, M. (2012). La televisión e Internet hoy: diferentes roles. Usos y consumos en el tiempo libre de jóvenes de Barcelona y Lima. Revista ICONO14, 10(3), pp. 176-201. Recuperado de http://doi.org/10.7195/ri14.v10i3.200

Fundamedios (2013). Nuevos públicos y tendencias en los medios de comunicación de Ecuador. Recuperado de http://files.ctctcdn.com/4d015e3d101/c0facf8d-5159-4b60ab15-a1053c318195.pdf

García, M. y Díaz, C. (2012). Radiografía del consumo de medios de comunicación en estudiantes universitarios. ICONO14. 10(3), pp. 100-115.

Gómez, A. (2015). Pew Research Center: "Hacer amigos por Internet es la nueva forma de socializar". Portal MediaTeleCom. Recuperado de http://mediatelecom.com.mx/index.php/tecnologia/item/90342-pew-research-centerhacer-amigos-por-Internet-es-la-nueva-forma-de-socializar

Gómez, L., López, N. y Martín, M. Á. (2011). Jóvenes, televisión y ocio digital. Cendi, 25.

INEC (2016). Ecuador en Cifras. Proyecciones poblacionales. Recuperado de http://www.ecuadorencifras.gob.ec/proyecciones-poblacionales/

Marín, I.; Yaguana, H. y Barrazueta P. (2013). Análisis del consumo de medios de comunicación en la juventud lojana de Ecuador. En Giménez, S. y Tarvido, G. (2013). 
Proyectos sociales, creativos y sostenibles (pp. 370-378). Toledo: Asociación Castellano Manchega de Sociología.

Marta, C.; Martínez, E. y Sánchez, L. (2013). La 'i-Generación’ y su interacción en las redes sociales. Análisis de Coca-Cola en Tuenti. Comunicar, XX(40), pp. 41-48.

Marth-Lazo, C. y Gabelas-Barroso, J. (2013). Hábitos de consumo televisivo de ficción entre los universitarios que estudian Comunicación. Revista de Comunicación de la SEECl, 31, pp. 14-33. Recuperado de http://doi.org/10.15198/seeci

Martínez, A. (2005). Consumption of television among youngsters at University. Comunicar, 25, pp. 1-8.

Masterman, L. (2010). La enseñanza de los medios de comunicación (Vol. 1). Madrid: Ediciones de la Torre.

Menor, J. (2010). Televisión, telefonía móvil y juventud en el contexto de la modernidad reflexiva de masas tardía: un proyecto de investigación. Revista de Estudios de Juventud, 88, pp. 25-50. Recuperado de http://doi.org/10.1016/j.chb.2001.01.019

MINTEL (2011). La Generación interactiva en Ecuador. Recuperado de http://es.scribd.com/doc/61621486/La-Generacion-Interactiva-en-Ecuador

Netlife (2015). Netlife analiza los hábitos digitales de los ecuatorianos. Recuperado de http://makrodigital.ec/?p=3084

Newman, N., et al. (2016). Reuters Institute Digital News Report 2016. Oxford: The Reuters Institute for the Study of Journalism.

Prensky, M. (2001). Digital natives, digital immigrants. On the Horizon, NCB University Press, 9(5). Recuperado de https://www.marcprensky.com/writing/Prensky\%20\%20Digital\%20Natives,\%20Digital\%20Immigrants\%20-\%20Part1.pdf

Rivière, M. (2003). El malentendido: como nos educan los medios de comunicación. Barcelona: Icaria Editorial.

Rubio, Á. (2010). Generación digital: patrones de consumo de Internet, cultura juvenil y cambio social. Revista de Estudios de Juventud, 88, pp. 201-221. Recuperado de http://doi.org/10.1177/009365001028004003

Sandoval, M. (2006). Los efectos de la televisión sobre el comportamiento de las audiencias jóvenes desde la perspectiva de la convergencia y de las prácticas culturales. Universitas Psychologica, 5(2), pp. 205-222.

Sesento, L. (2015). La influencia de los medios de comunicación en los jóvenes, Revista Contribuciones a las Ciencias Sociales, 29. Recuperado de http://www.eumed.net/rev/cccss/2015/03/informacion-jovenes.html

TtvNews (2014). Los jóvenes se alejan de la TV tradicional. Recuperado de http://www.todotvnews.com/news/estudiotvtradicionalviejos.html 
Vergara, M. et al. (2009). Consumo de medios masivos de comunicación en estudiantes universitarios de Manizales. Hacia La Promoción de La Salud, pp. 124-138. Recuperado de http://www.scielo.org.co/scielo.php?script=sci_arttext\&pid=S0121$75772009000100009 \&$ lang=pt

Zamora, J. (2004). Medios de comunicación. Información, espectáculo, manipulación. Madrid: Editorial Verbo Divino.

Zuberogoitia, A. et al. (2016). Consumo de series de televisión de los adolescentes en la era de la digitalización audiovisual: prácticas y motivaciones. Dígitos, 2, pp. 35-52. Recuperado de http://roderic.uv.es/handle/10550/53916 